\title{
A PRÁTICA PEDAGÓGICA DA INTEGRAÇÃO DE TECNOLOGIAS CONSTRUTIVAS COMO FERRAMENTA DE PROJETO
}

\section{The pedagogical practice of the integration of construction} technologies as a design tool

\author{
Luis Henrique Alves Cândido', Suzane Miritz', Jéssica do Canto Pureza'
}

RESUMO Das etapas metodológicas para o desenvolvimento de produtos, a aplicação de práticas construtivas pode ser orientada pela fabricação de modelos, protótipos e maquetes. Diante da evolução das ferramentas de projeto e da necessidade contínua de inovação, cada vez mais se necessita de laboratórios e oficinas que contemplem estruturas tecnológicas de equipamentos e pessoal de apoio. Essa estrutura visa a auxiliar a prática pedagógica orientada pela integração do projeto virtual e sua análise física. Nesse sentido, este trabalho tem por objetivo apresentar um estudo de caso no qual foi desenvolvido um equipamento utilizado para filtragem de água para consumo humano. Para isso, foi realizada a construção de um modelo estrutural que integrou tecnologias de corte a laser, de impressão 3D e processos manuais de fabricação. O foco dessa prática foi a análise, a reflexão e a validação de princípios e parâmetros projetuais a serem aplicados no projeto final. O resultado da aplicação dessa prática pedagógica, apontam para a relevância da integração de processos tecnológicos com práticas manuais, proporcionando, dessa forma, um fluxo contínuo e dinâmico de aprendizagem orientada para a resolução de problemas funcionais e construtivos.

\section{PALAVRAS-CHAVE: Modelos, Tecnologias, Integração.}

ABSTRACT From methodological stages to product development, the application of construction practices may be oriented for the manufacturing of models, prototypes and scale models. Upon the evolution of design tools and the continuous need for innovation, laboratories and workshops that address equipment technological infrastructures and support staff are increasingly needed. This framework aims to assist the pedagogical practice guided by the integration of virtual project and its physical analysis. In this sense, this work presents a case study in which a device used for water filtration for human consumption was developed. For this purpose, the construction of a structural model that integrated laser-cutting technologies, 3D printing and manual manufacturing processes was performed. Analyzing, reflecting and validating the principles and project parameters to be applied in the final design was the focus of this practice. The result of this pedagogical practice points to the importance of the integration of technological processes with manual practices, thus providing a continuous and dynamic flow of learning-oriented constructive resolution of functional problems.

KEYWORDS: Models, Technologies, Integration.

\section{How to cite this article:}

CÂNDIDO, L. H. A.; MIRITZ S.; PUREZA, J. C. Gestão e Tecnologia de Projetos, São Carlos, v. 11, n. 1, p. 39-53, jan./jun. 2016.http://dx.doi.org/10.11606/gtp.v11i1.99133 


\section{INTRODUÇÃO}

O designer vem, ao longo do tempo, garantindo um papel fundamental no processo de criação de produtos. Existem técnicas que auxiliam no direcionamento do caminho a seguir, e as metodologias de desenvolvimento de produto são ferramentas essenciais diante de um mercado tão concorrido e restrito. Elas são essenciais para o processo de design no momento em que traçam diretrizes para o desenvolvimento de produtos e caracterizam-se por estudos de princípios e procedimentos fortemente orientados. Uma das práticas apresentadas na maioria das metodologias projetuais, segundo seus autores (ABRAMOVITZ, 2002; BAXTER, 2000; BACK et al., 2008; BOOTHROYD; ALTING, 1992; BITTENCOURT, 2001; BOMFIM, 1995; BONSIEPE, 1984; LÖBACH, 2000; ROOSEMBURG; EEKELS, 1996; PLATCHECK, 2012), consiste na aplicação e construção de modelos e protótipos, físicos e virtuais.

Conforme Santos (2005), modelos e protótipos físicos são fundamentais para o desenvolvimento de produtos, para o planejamento da produção, do setup de máquinas, da avaliação do layout fabril e para ensaios de desempenho do produto. Essa prática permite ao profissional das áreas de projeto a análise e avaliação do objeto em estudo, como a forma do produto, as cores aplicadas, a textura, o acabamento, os detalhes, a funcionalidade e as adequações ergonômicas. Os modelos e protótipos, segundo Santos (2005), podem ser empregados na pesquisa sobre experiências de uso e determinar a continuação de um projeto, sua alteração ou total abandono. Ainda segundo o mesmo autor, as informações transmitidas por meio de um modelo ou protótipo físico, se comparadas a modelos virtuais, tendem a ser melhor assimiladas pela maioria das pessoas. Essa facilidade na assimilação se deve ao fato de que por meio dos objetos físicos várias percepções do ser humano são estimuladas.

Segundo Florio (2011), a integração do processo criativo a práticas construtivas demanda um forte envolvimento do professor no sentido de mostrar e demonstrar o potencial dessa integração nas etapas de soluções de projeto. Segundo Pertence et al. (2001), a absorção tridimensional se desenvolve à medida que um indivíduo vivencia o espaço, principalmente por meio da visão e do tato, que são responsáveis por captar estímulos, como brilho, sombra, cor, frio, calor e outros. Esses estímulos são processados pelo cérebro, que assim elabora a forma, a proporção, a posição e a orientação do produto no espaço. Após essa etapa, o cérebro compara, classifica e interpreta essa nova informação com modelos observados anteriormente.

Forti (2005) descreve que os modelos e protótipos tridimensionais físicos trazem diversas vantagens para o ambiente de projeto, isso porque anulam o esforço cognitivo de se interpretar palavras ou imagens totalmente bidimensionais. Conforme escrito em Alvares (2004), Hans Gugelot, na década de 1960, desenvolveu uma metodologia de projeto com uma sequência de atividades projetuais básicas para o design de produtos industriais, e que atualmente serve de base para os cursos de design. Essa proposta consiste nas seguintes fases: fase de informação, fase analítica, fase de projeto, fase de decisão, fase de cálculos e adaptações e fase da construção de modelos e protótipos.

Mesmo que essa nomenclatura mude em alguns currículos, o objetivo é o mesmo, isto é, proporcionar a compreensão do processo de elaboração física de um produto. Em muitos casos, os tipos de materiais e processos apresentados ficam restritos à estrutura física de cada instituição, ou seja, para cada tipo de material são necessários processos e equipamentos específicos. Os processos de fabricação são aplicados quando os materiais precisam adquirir formas e dimensões para ser utilizáveis na indústria, e são definidos também em função das propriedades dos materiais e das características necessárias para resistir às condições de serviço da peça.

Logo, ao trabalhar com os materiais na forma de modelos e protótipos, busca-se proporcionar ao aluno um contato físico com esses materiais, e assim demonstrar as variáveis que estes podem apresentar. Conforme Ashby 
e Johnson (2003), os produtos alcançam sucesso com uma combinação entre o bom projeto técnico e o projeto industrial criativo, no qual os materiais e os processos são usados para fornecer a funcionalidade, a usabilidade e a satisfação na compra. A satisfação é extremamente influenciada pela estética do produto, pelas associações que o usuário faz no momento da compra e pelas percepções, muitas vezes intuitivas, que o produto transmite ao usuário.

\section{CONSTRUÇÃO DOS MODELOS E PROTÓTIPOS}

No desenvolvimento de projetos inovadores em design e arquitetura, a utilização de modelos, protótipos e maquetes visa, principalmente, à verificação e validação das soluções geradas nas representações bidimensionais e virtuais. A necessidade de construir modelos e protótipos está no fato de permitir ao acadêmico a análise e validação do objeto em estudo, tanto na sua forma, nas cores, nas texturas, nos acabamentos, na funcionalidade e na adequação técnica e normativa. Assim, algumas técnicas, conforme Volpato (2007), podem ser aplicadas para construção de elementos físicos e, dentre essas, encontram-se as seguintes:

a) modelo volumétrico - pode ser executado em diversos tipos de materiais, sem a necessidade de relação com o do produto final;

b) protótipo ou mock-up - reproduzem fielmente o objeto final a ser produzido em série e servem para avaliar o desempenho do objeto projetado na sua utilização normal;

c) modelo operacional e modelo estrutural - corresponde às etapas de avaliação da funcionalidade, podendo, aplicar mecanismos como engrenagens, transmissão de movimentos, encaixes, subsistemas de fixação e outros.

Outro aspecto que podemos abordar na construção de modelos e protótipos diz respeito à ética na etapa da seleção da melhor alternativa. A ética, segundo Alvares (2004), étratada, também, na grade curricular de cursos de Design, sendo balizadora de princípios que orientam o comportamento social, fato que deve ser avaliado na fabricação de projetos. Esses princípios caminham por uma linha muito tênue que fica entre a discussão sobre princípios de design e da experiência dos agentes envolvidos. Então, observase que em uma sociedade industrial onde o mundo tecnológico tem causado grandes conflitos, principalmente coletivos, deve-se levar em consideração a vulnerabilidade da natureza do criador do projeto, ou seja, o designer pode sentir que sua criação supre todas as necessidades na função requerida, sem ao menos verificar todas as possíveis soluções.

Sob essa perspectiva, ao utilizarmos a tecnologia apropriada para o ensino didático da construção de modelos e protótipos, poderemos discutir várias alternativas de forma compartilhada, ou seja, é possível observar e compreender a evolução do projeto virtual até a escolha da melhor alternativa. Assim, podemos inferir que a integração da ética e da tecnologia torna o processo de ensino de projeto mais transparente, principalmente por considerar parâmetros de escolha projetuais orientados por ensaios físicos e funcionais.

Neste sentido, este artigo apresenta o resultado de um estudo de caso que utiliza a tecnologia de corte a laser e impressão 3D para a discussão didática sobre a seleção da melhor alternativa projetual. Logo, o artigo propõe que ao utilizar a tecnologia construtiva no processo de ensino de projeto, podese ampliar a discussão e entendimento do acadêmico de design sobre os motivos concretos que balizaram a escolha da alternativa final. Além da questão didática abordada, busca-se compartilhar o conhecimento científico 
adquirido por meio da apresentação de parâmetros de corte, levantados em materiais de grande utilização para fabricação de modelos e protótipos, e as relações de tempo de fabricação via corte laser e impressão 3D.

\section{Laboratório de modelos, protótipos e maquetes}

A utilização de um laboratório de modelos, protótipos e maquetes durante as disciplinas de projeto tende a possibilitar a integração dos esboços iniciais, em papel ou virtuais, com os modelos e protótipos físicos. Esse processo pedagógico proporciona aos acadêmicos a oportunidade de percorrer e acompanhar as transformações e as contribuições que a prática construtiva pode aportar ao projeto final. Isso ocorre porque podemos integrar simultaneamente os materiais, os processos de fabricação e as soluções mecânicas e discutir conceitos estéticos. Para a perfeita integração dessas variáveis, é fundamental a introdução de equipamentos que possibilitem a modelagem de um produto e seus sistemas o mais fielmente possível.

Diante da evolução tecnológica desses equipamentos, fica evidente, também, sua influência no processo de criação de produtos. Nossa experiência na utilização desses equipamentos, por acadêmicos de design, de arquitetura e urbanismo, demonstrou claramente uma evolução no processo de projetação. Como exemplo, devido à inserção da máquina de corte a laser, os projetos nessas áreas tornaram-se mais complexos e com maior nível de detalhamento.

Observou-se que anteriormente à instalação do equipamento, o processo de criação era orientado fortemente pelo corte manual e, dessa forma, poderia ocorrer um limite criativo devido à restrição desse processo de corte. Porém, com a introdução da primeira máquina de corte a laser, o processo de criação ficou mais apurado. Dessa forma, podemos inferir que a criação não pode depender da técnica, mas a técnica pode e deve auxiliar na evolução do processo criativo. Diante dessa reflexão, são apresentados na Figura 1 alguns equipamentos que fazem parte da estrutura do Laboratório de Modelos, Protótipos e Maquetes da Faculdade de Arquitetura (FA/UFRGS).

Figura 1. Porta de entrada da oficina (Figura 1A), máquina de corte a laser 200 watts (Figura 1B), e máquina de corte a laser 60 watts (Figura 1C) e impressoras 3D (Figura 1D).
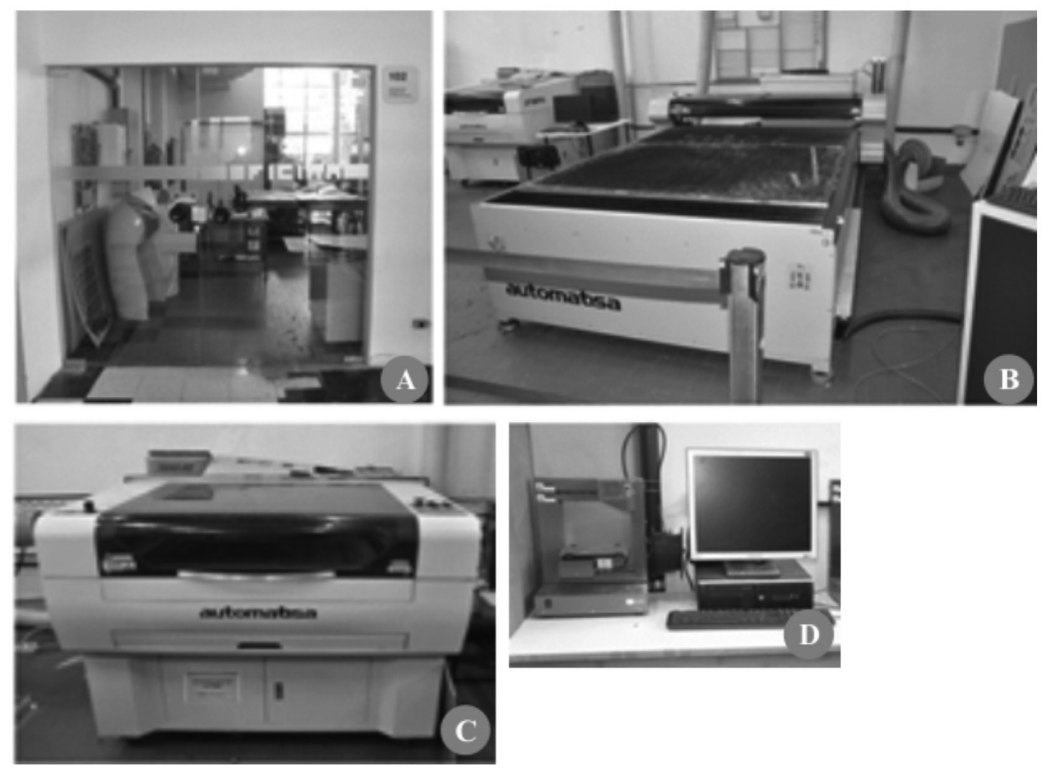

A máquina de corte a laser de 200 watts (modelo ACRILA, Figura 1B) proporciona um corte máximo de $1500 \mathrm{~mm} \times 2500 \mathrm{~mm}$. A máquina de corte a laser de 60 watts (modelo PRISMA, Figura 1C) proporciona um corte 
máximo de $600 \mathrm{~mm} \times 900$ mm. A impressora 3D (marca CLIEVER, Figura 1D) foi utilizada para construção de modelos volumétricos visando a validação da seleção das propostas de peças estruturais. A impressora possibilita a construção de objetos com área máxima de $100 \mathrm{~mm} \times 100 \mathrm{~mm} \times 100 \mathrm{~mm}$. Para o presente trabalho, a operação de corte a laser foi balizada com o uso da máquina de 60 watts.

A oficina (Figura 1A) fica aberta durante o horário das aulas dos cursos de Arquitetura, Urbanismo, Design de Produto e Design Visual. A estrutura atende a esses cursos de graduação e de pós-graduação oriundos da Faculdade de Arquitetura como o PGDESIGN, PROPAR e PROPUR. O atendimento a acadêmicos e professores é realizado com o apoio de técnicos que ficam à disposição na oficina, que têm a função de operar os equipamentos de fabricação, como a máquina de corte a laser e equipamentos de pequeno porte.

\section{MÉTODO DA PESQUISA}

\section{Contexto metodológico}

O estudo de caso foi desenvolvido a partir da temática de um projeto de produto voltado para soluções de purificação de águas contaminadas. O delineamento do trabalho é orientado pela pesquisa experimental, que visou a demonstrar a integração de ferramentas tecnológicas no processo de construção funcional de modelos e protótipos, com o ensino de design de produto, mais especificamente sobre a discussão objetiva da escolha da melhor alternativa de projeto.

Segundo Prodanov (2009), a pesquisa experimental é mais frequentemente empregada nas ciências tecnológicas e tem como objetivo demonstrar como e por que um determinado fato pode ser produzido.

Historicamente, a utilização de modelos e protótipos para o desenvolvimento de produtos talvez seja uma das mais clássicas disciplinas que acompanham o processo de design. Assim, diversas técnicas foram desenvolvidas e são adotadas em cursos de design no Brasil. Porém, é possível observar que alguns desses processos tornaram-se pouco eficientes devido à necessidade de sua aplicação em produtos inovadores e com uma alta complexidade construtiva.

Certamente, a aplicação de técnicas clássicas de modelagem continua fundamental na prática didática do ensino de Design; porém, o dinamismo e o acesso a equipamentos tecnológicos de fabricação agilizaram a tomada de decisão sobre a melhor estrutura funcional do produto a ser desenvolvido. Dessa forma, ao utilizar técnicas de rápida aplicação como o corte a laser aliado à geração de alternativas construtivas, poderemos acelerar a tomada de decisão objetiva sobre a melhor solução funcional do produto. A seguir, a Figura 2 apresenta o delineamento do processo de pesquisa orientador desse trabalho.

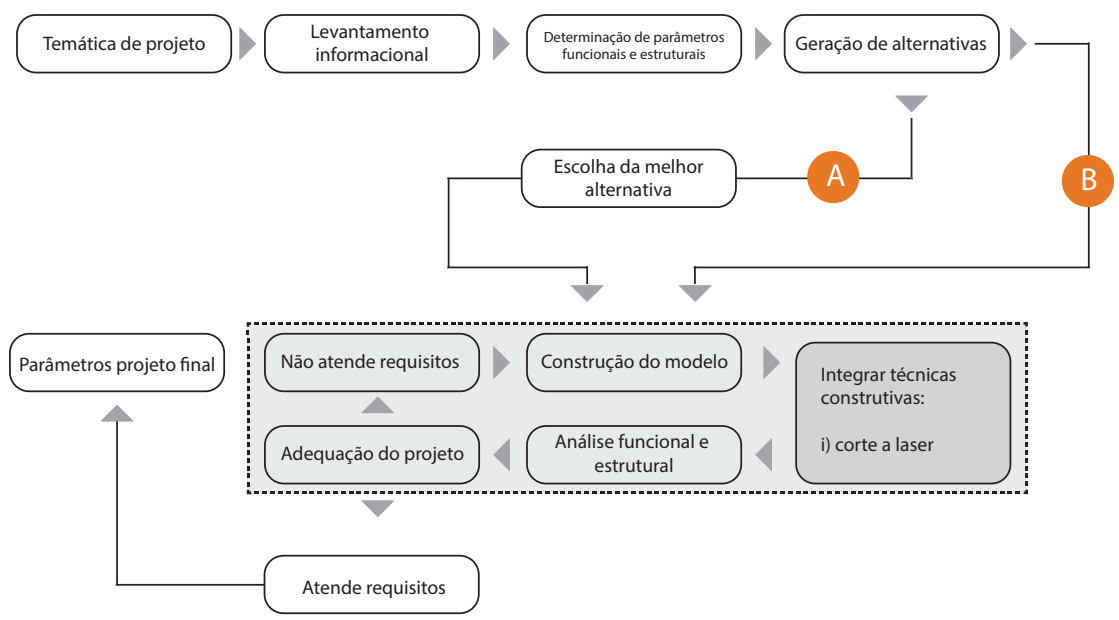

Figura 2. Fluxograma de aplicação didática. 
A Figura 2 apresenta o fluxograma do processo de modelagem, que destaca a integração de técnicas construtivas ao processo de adequação do projeto. Verifica-se que se o projeto não atender ao parâmetro funcional e estrutural pré-determinado, ele volta para a etapa de construção do modelo, utilizando, para isso, a integração das técnicas até que essas melhorias se equalizem com os parâmetros de projeto. No momento em que o projeto atende aos requisitos, segue para a solução final. Cabe ressaltar que mesmo entrando nessa etapa o projeto poderá retornar para a construção do modelo, caso surjam dúvidas funcionais, estruturais ou estéticas.

O caminho "A" apresentado no fluxograma representa o modo clássico da escolha da melhor alternativa de projeto, ou seja, as alternativas geradas são classificadas e reduzidas a um número julgado ideal pela equipe de projeto. Porém, o caminho "B", sugerido nessa pesquisa, amplia o número de soluções possíveis de serem fabricadas, ou seja, a de testar múltiplas alternativas.

Durante o desenvolvimento de um projeto, a etapa da tomada de decisão sobre a seleção de alternativas pode ser considerada uma das mais relevantes para o sucesso do produto. Sendo assim, o aprofundamento na etapa de seleção de conceitos deve ser associado a uma maior quantidade e qualidade de informações, reduzindo, dessa forma, os riscos de uma tomada de decisão equivocada.

Segundo Back et al. (2008), os problemas na tomada de decisão da melhor alternativa podem ser classificados em duas vertentes. A primeira vertente sugere a tomada de decisão por um viés subjetivo e sem critérios bem definidos, com forte influência pessoal. A segunda vertente ocorre devido à falta de um método específico e a não consideração da integração entre critérios de seleção. $O$ fator de influência pessoal para escolha da melhor alternativa, segundo descrito por Camargo (2007), é considerado um importante fato para o insucesso do produto final, e deve ser evitado.

Camargo (2007) descreve que o processo de decisão deve considerar fatores como demandas do cliente, parâmetros de engenharia e o foco competitivo da empresa, sendo estes associados às ferramentas técnicas para a avaliação de alternativas. Diante do exposto, devemos, durante o processo didático do ensino de projeto, considerar a minimização de tais fatos que podem levar o acadêmico a não compreender claramente como foi tomada a decisão sobre a escolha da melhor alternativa.

A falta de uma maior clareza sobre essa etapa pode, no futuro, representar o insucesso de um produto e o insucesso do profissional. Dessa forma, devemos utilizar técnicas construtivas que possibilitem a análise dos aspectos funcionais e estruturais de um produto de forma a orientar, com maior objetividade, e não subjetividade, a escolha da melhor alternativa durante o processo de ensino.

A experiência, por meio dessa prática construtiva, aponta para algumas tecnologias que possibilitam uma rápida aplicabilidade para solução de projetos. Assim, pode-se apontar que primeiramente temos o corte a laser e, em segundo patamar, a impressão 3D. Isso significa que com auxílio da tecnologia de corte a laser, para construção de componentes e estruturas funcionais de forma plana, teremos uma maior rapidez na fabricação desses elementos em relação à impressão 3D, que nesse caso leva-se muito tempo para a fabricação final da peça.

O fluxograma apresentado na Figura 2 "caminho "B” busca potencializar o processo de ensino de projeto, pois possibilita um fluxo contínuo de aprimoramento da proposta, ou seja, a dinâmica ocorre no momento em que empregamos, para fabricação dos modelos, técnicas de rápida construção, como o corte a laser. Essa prática proporciona ao acadêmico uma maior imersão na problemática de projeto, e assim as soluções podem ser construídas e testadas de forma mais rápida em relação à aplicação de técnicas de impressão 3D. 
A integração apresentada no fluxograma (Figura 2, caminho B) visa a potencializar o maior entendimento sobre os requisitos funcionais e estruturais do produto. Assim, ao integrar um maior número de alternativas ao processo de fabricação física do modelo poderemos ampliar o espectro acadêmico da aprendizagem orientado por fatores objetivos durante as etapas de geração e seleção de alternativas projetuais.

\section{Geração de alternativas projetuais}

A temática do estudo de caso é a concepção de um produto que visa à purificação de águas extremamente contaminadas e impróprias para consumo. O projeto apresenta a problematização da ausência de água potável em diversos continentes, onde é necessária a existência de um equipamento que purifique e forneça água em grande volume. Outro requisito de projeto apresenta a necessidade de transporte do produto ao local de filtragem da água. Neste sentido, a Figura 3 apresenta a estrutura funcional do produto e os módulos integrantes.

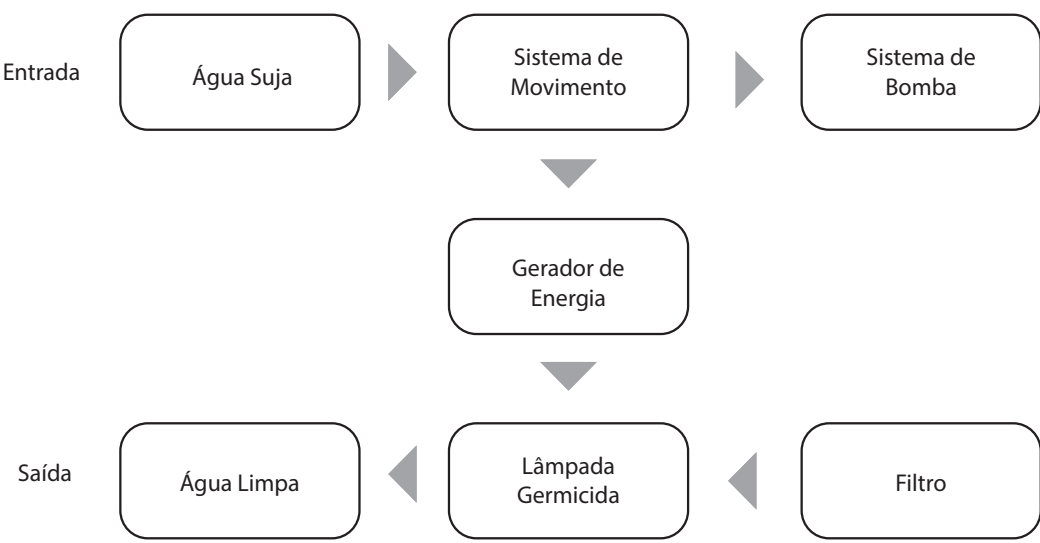

A partir do diagrama funcional apresentado na Figura 3 foram geradas alternativas de projeto, e posteriormente a construção de um protótipo funcional, tendo como foco ampliar a compreensão funcional do produto. Assim, foram utilizados diversos materiais para sua construção como tubos e conexões de PVC, MDF, parafusos, filtro à vela industrial, lâmpada germicida (15 watts) e componentes mecânicos oriundos da desmontagem de uma bicicleta. Diante da temática inicial foram desenvolvidas soluções de projeto empregando, para isso, a técnica do esboço via sketches.

Seguindo a sequência de desenvolvimento do projeto, a etapa posterior consistiu na geração de alternativas de mecanismos que integrassem princípios mecânicos de baixa manutenção e eficiência na utilização. Assim, a Figura 4 mostra os princípios de projeto gerados em 2D.

Na Figura 4A observa-se o sistema completo; na Figura 4B observa-se o sistema de movimento vertical da bomba; na Figura 4C observa-se o sistema hidráulico; e na Figura 4D vê-se o sistema de fixação do pedal de acionamento da bomba. Orientado por essas alternativas, foram selecionados, para pratica construtiva, os sketches apresentados na Figura 5 e na Figura 6.

A Figura 5 apresenta a geração de dez alternativas de apoio para o pedal, a Figura 5A sendo a primeira alternativa e a Figura 5J a alternativa utilizada no projeto final. A Tabela 1 descreve a dimensão retangular balizadora das peças apresentadas na figura 5 .

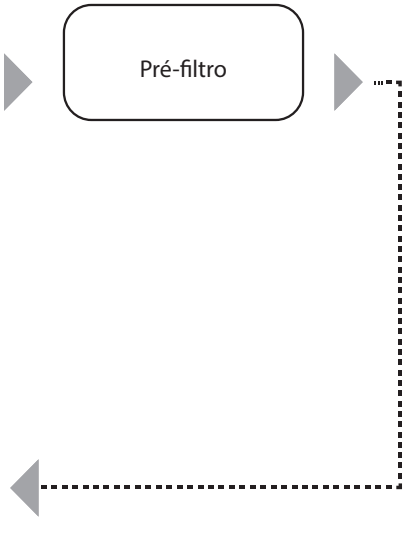

Figura 3. Diagrama funcional. 
Figura 4. Geração de alternativas 2D.
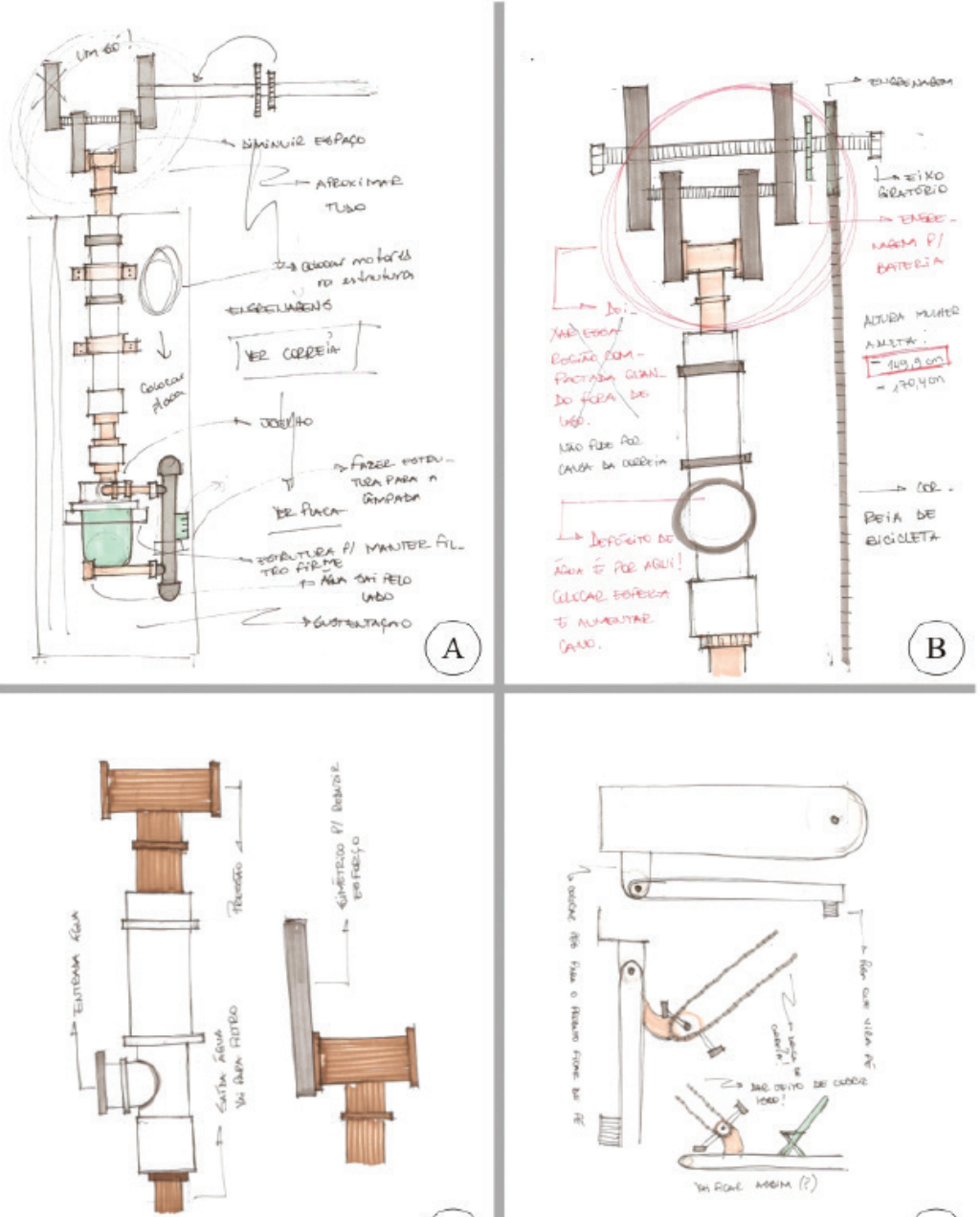

(C)

(D)

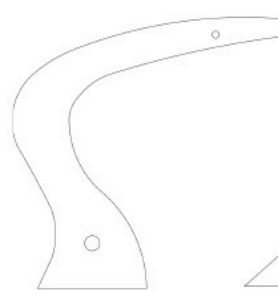

A

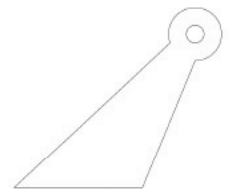

F

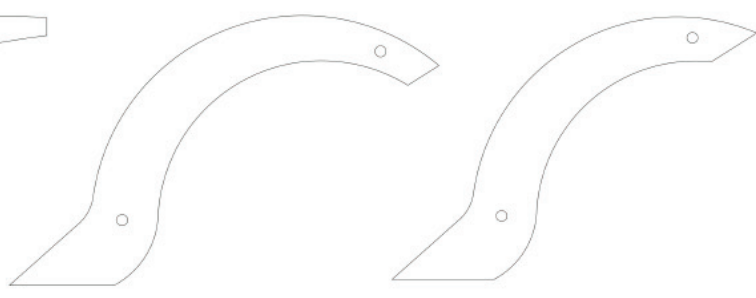

B
$\mathrm{C}$

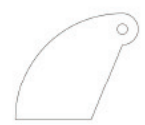

$\mathrm{H}$

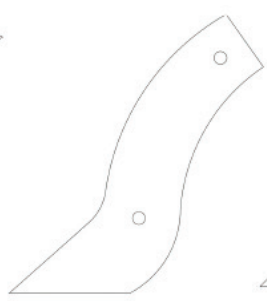

D

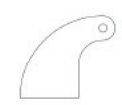

I

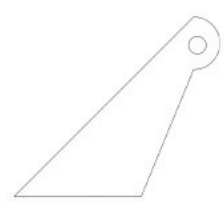

E

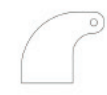

J

Figura 5. Geração de alternativas do apoio do pedal. 
Tabela 1. Parâmetros dimensionais para corte do apoio do pedal.

\begin{tabular}{cccccccccccccc}
\hline \multicolumn{10}{|c|}{ Figura 5 } & & & & \\
\hline Peça & A & B & C & D & E & F & G & $H$ & I & J \\
$\begin{array}{c}\text { Dimensões } \\
(\mathrm{mm})\end{array}$ & $515 \times 540$ & $817 \times 515$ & $694 \times 500$ & $485 \times 531$ & $388 \times 342$ & $393 \times 342$ & $298 \times 255$ & $238 \times 204$ & $179 \times 159$ & $152 \times 140$ \\
\hline
\end{tabular}

A Figura 6 apresenta as alternativas para a fabricação da engrenagem.

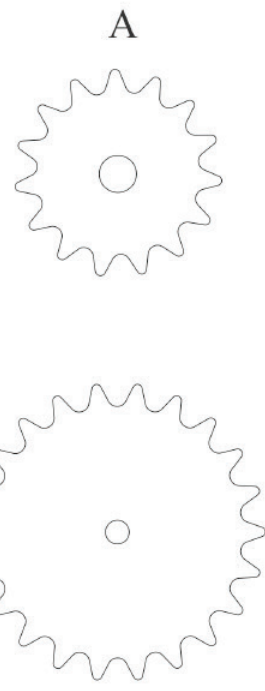

$\mathrm{E}$
B
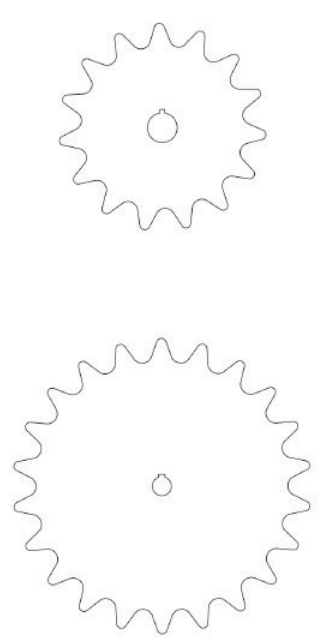

$\mathrm{F}$
$\mathrm{C}$
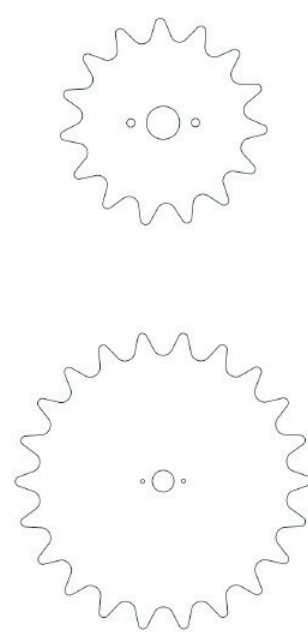

G
$\mathrm{D}$
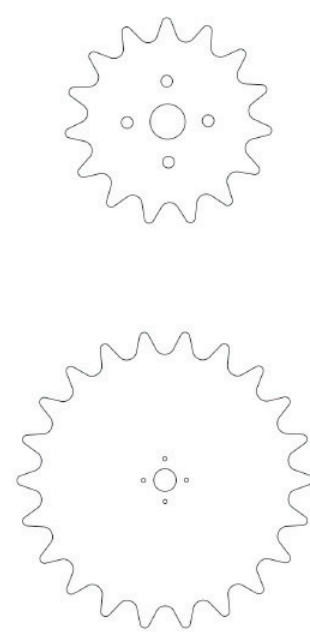

$\mathrm{H}$

Figura 6. Geração de alternativas de engrenagens para a bomba de água. tipos de fixação, sendo que a engrenagem da Figura 6D foi a escolhida para inclusão no projeto final. A próxima etapa da pesquisa levantou os parâmetros de corte de alguns dos principais materiais utilizados no design para construção de modelos e protótipos. Esse levantamento foi balizador para a seleção do material a ser utilizado para fabricação das peças das Figuras 5 e 6. Assim, a Tabela 2 apresenta os parâmetros de potência aplicados nos respectivos materiais, sendo cortados na máquina de 60 watts. Os parâmetros foram gerados a partir de um corte padrão de dimensão retangular de $40 \mathrm{~mm} \times 40 \mathrm{~mm}$.

A Tabela 2 apresenta uma série de parâmetros de corte e uma diversidade de materiais que são utilizados para a fabricação de modelos e protótipos. Destacam-se os materiais grifados que não atingiram o corte completo da peça mesmo aplicando $100 \%$ da potência de corte da máquina. Diante dos parâmetros levantados, optou-se pela construção das peças (da Figura 5 e da Figura 6) utilizando-se o MDF. A seleção levou em consideração a necessidade estrutural e funcional dessas peças ao serem ensaiadas fisicamente no produto final.

A etapa seguinte focou o levantamento dos parâmetros de profundidade de corte, ou seja, a penetração do laser no material. Essa etapa foi realizada utilizando como material base o MDF na espessura de $8 \mathrm{~mm}$. Os dados levantados são apresentados no Gráfico 1. 
Tabela 2. Parâmetros de corte de máquina laser 60 watts.

\begin{tabular}{|c|c|c|c|}
\hline Material & Espessura & $\begin{array}{l}\text { Potência de } \\
\text { corte (watts) }\end{array}$ & $\begin{array}{c}\text { Velocidade de corte } \\
(\mathrm{m} / \mathrm{min})\end{array}$ \\
\hline \multirow{7}{*}{$\begin{array}{l}\text { MDF } \\
\text { (Medium Density } \\
\text { Fiberboard) }\end{array}$} & 3 & 100 & 1 \\
\hline & 4 & 100 & 1 \\
\hline & 5 & 100 & 1 \\
\hline & 8 & 100 & 0,5 \\
\hline & 9 & 100 & 0,5 \\
\hline & 12 & 100 & 0,5 \\
\hline & 15 & 100 & 0,5 \\
\hline $\begin{array}{l}\text { OSB (Oriented Strand } \\
\text { Board) }\end{array}$ & 10 & 100 & 0,5 \\
\hline Compensado naval & 10 & 100 & 0,5 \\
\hline Madeira balsa & 2 & 80 & 45 \\
\hline \multirow{6}{*}{ Acrílico cristal } & 2 & 80 & 20 \\
\hline & 3 & 80 & 20 \\
\hline & 4 & 100 & 15 \\
\hline & 5 & 100 & 5 \\
\hline & 6 & 100 & 5 \\
\hline & 10 & 100 & 1 \\
\hline Acrílico branco & 2 & 80 & 20 \\
\hline \multirow{2}{*}{ Acetato } & 0,5 & 100 & 5 \\
\hline & 0,8 & 100 & 5 \\
\hline Poliestireno & 5 & 20 & 100 \\
\hline Cortiça & 2 & 10 & 100 \\
\hline \multirow{2}{*}{ Papelão três camadas } & 2,5 & 80 & 50 \\
\hline & 6 & 80 & 50 \\
\hline Papel pluma & 5 & 10 & 90 \\
\hline Couro sintético & 0,5 & 20 & 80 \\
\hline \multirow{2}{*}{ Papel hurley } & 1 & 50 & 35 \\
\hline & 2 & 30 & 30 \\
\hline \multirow{2}{*}{ Papel cartão } & 2 & 50 & 30 \\
\hline & 2,5 & 50 & 30 \\
\hline Papel Duplex & 2 & 50 & 80 \\
\hline Papel Triplex & 0,5 & 60 & 70 \\
\hline
\end{tabular}

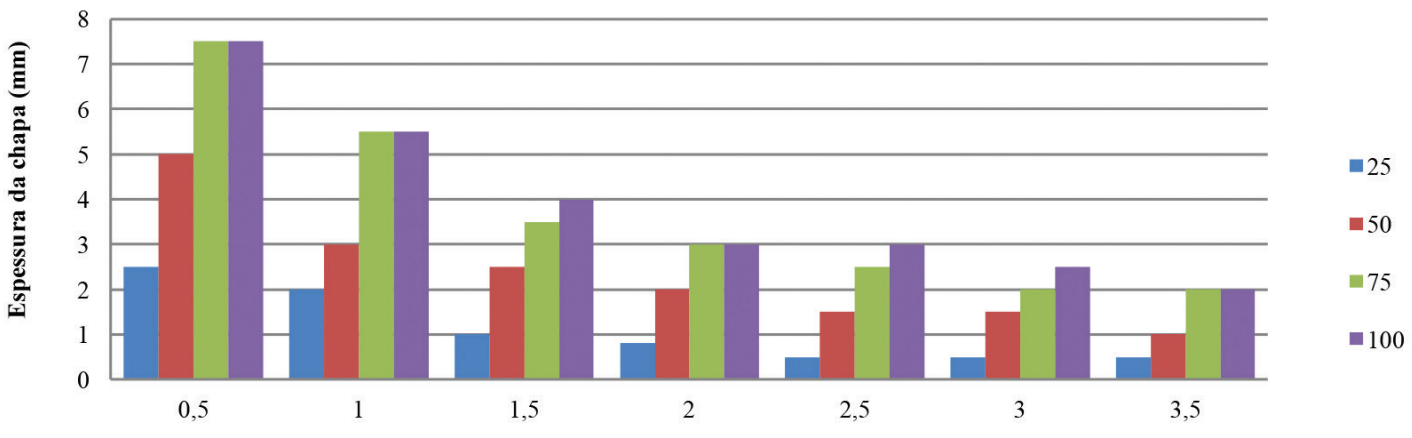

Velocidade (m/min)

Gráfico 1. Parâmetros de

profundidade de penetração do laser. 
No Gráfico 1 observa-se que a velocidade de corte variou de 0,5 até 3,5 ( $\mathrm{m} / \mathrm{min})$ da velocidade total da máquina. Os fatores de potência foram determinados entre $25 \%, 50 \%, 75 \%$ e $100 \%$ da potência. O levantamento desses parâmetros agiliza a etapa de transferência do sketch virtual para a construção física da peça. Assim, a pré-qualificação de quais parâmetros utilizarem é determinada antecipadamente. Diante dos dados obtidos, os seguintes parâmetros foram utilizados para o corte final das peças (das Figuras 5 e 6): espessura do MDF de $5 \mathrm{~mm}$, velocidade de corte de $1 \mathrm{~m} / \mathrm{min}$ e potência de corte de $75 \%$. Esses parâmetros possibilitam o corte efetivo das peças sem utilizar a potência de $100 \%$ da máquina. Dessa forma, busca-se ampliar a vida útil do equipamento e a redução do consumo de energia. Embora a velocidade de corte de $0,5 \mathrm{~m} / \mathrm{min}$ possa ser utilizada, essa configuração gerou uma queima excessiva na aresta do MDF, marcando o contorno da peça.

Visando a levantar os dados comparativos entre o corte a laser e a impressão 3D, as peças da Figura 6 foram construídas também via impressão 3D. As peças da Figura 5 não foram construídas com esse processo devido às dimensões externas ultrapassarem a capacidade máxima da mesa de apoio da impressora. Assim, a Tabela 3 apresenta o levantamento do tempo de corte das peças desenvolvidas e apresentadas nas Figuras 5 e 6 .

Tabela 3. Parâmetros de fabricação para as peças das Figuras 5 e 6.

\begin{tabular}{|c|c|c|c|c|}
\hline Peça & $\begin{array}{l}\text { Potência de corte } \\
\text { do laser } \\
(\%)\end{array}$ & $\begin{array}{l}\text { Velocidade de } \\
\text { corte } \\
(\mathrm{m} / \mathrm{min})\end{array}$ & Tempo de corte & Tempo de impressão (3D) \\
\hline Figura 5 - A & \multirow{10}{*}{75} & \multirow{10}{*}{1} & $02 \mathrm{~min} 20 \mathrm{seg}$ & \multirow{10}{*}{$\begin{array}{l}\text { Não aplicável devido à } \\
\text { restrição dimensional da } \\
\text { mesa de impressão }\end{array}$} \\
\hline Figura 5 - B & & & $02 \mathrm{~min} 31 \mathrm{seg}$ & \\
\hline Figura 5 - C & & & $02 \mathrm{~min} 13 \mathrm{seg}$ & \\
\hline Figura 5 - D & & & $01 \mathrm{~min} 54 \mathrm{seg}$ & \\
\hline Figura 5 - E & & & $01 \mathrm{~min} 24 \mathrm{seg}$ & \\
\hline Figura 5 - F & & & $01 \mathrm{~min} 25 \mathrm{seg}$ & \\
\hline Figura $5-\mathrm{G}$ & & & $01 \mathrm{~min} 06 \mathrm{seg}$ & \\
\hline Figura $5-\mathrm{H}$ & & & $00 \mathrm{~min} 53 \mathrm{seg}$ & \\
\hline Figura 5 - I & & & 00 min 42 seg & \\
\hline Figura $5-\mathrm{J}$ & & & $00 \mathrm{~min} 38 \mathrm{seg}$ & \\
\hline Tempo total de corte & & & $15 \mathrm{~min}$ e $10 \mathrm{seg}$ & 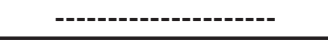 \\
\hline Figura 6 - A & \multirow{8}{*}{75} & \multirow{8}{*}{1} & $00 \mathrm{~min} 16 \mathrm{seg}$ & 01 hora 39 min \\
\hline Figura 6 - B & & & $00 \mathrm{~min} 16 \mathrm{seg}$ & 01 hora $39 \mathrm{~min}$ \\
\hline Figura 6 - C & & & $00 \mathrm{~min} 21 \mathrm{seg}$ & 01 hora $45 \mathrm{~min}$ \\
\hline Figura 6 - D & & & $00 \mathrm{~min} 22 \mathrm{seg}$ & 01 hora $42 \mathrm{~min}$ \\
\hline Figura $6-E$ & & & $00 \mathrm{~min} 25 \mathrm{seg}$ & 03 horas $20 \mathrm{~min}$ \\
\hline Figura 6 - F & & & $00 \mathrm{~min} 25 \mathrm{seg}$ & 03 horas $23 \mathrm{~min}$ \\
\hline Figura $6-G$ & & & $00 \mathrm{~min} 27 \mathrm{seg}$ & 03 horas 23 min \\
\hline Figura $6-\mathrm{H}$ & & & $00 \mathrm{~min} 29 \mathrm{seg}$ & 03 horas 24 min \\
\hline \multicolumn{3}{|l|}{ Tempo total de corte } & $3 \mathrm{~min}$ e $01 \mathrm{seg}$ & 20 horas e $25 \mathrm{~min}$ \\
\hline
\end{tabular}

A Tabela 3 apresenta informações que são fundamentais no processo de fabricação de peças, principalmente, o tempo de fabricação. Dessa forma, ao escolher o processo adequado de fabricação, poderemos enriquecer a discussão objetiva sobre a avaliação de alternativas, isso porque temos a condição de fabricar componentes complexos e com baixo tempo de processamento.

Observa-se na Tabela 3 que a relação de tempo entre o processo de corte a laser e a impressão 3D para a fabricação da peça 6 é substancialmente diferente. Se a opção da construção dessa peça fosse orientada pela fabricação 3D, o tempo total seria elevado em mais de 20 horas, enquanto o 
Figura 7. Construção do modelo funcional da bomba e filtro. corte via tecnologia laser consumiria um pouco mais de 3 minutos. Assim, fica claro que a correta utilização do processo de fabricação visa possibilitar uma maior imersão, de acadêmicos e professores, na etapa de discussão sobre as melhores propostas de projeto, durante a construção do protótipo.

Visando avaliar fisicamente as peças fabricadas das Figuras 5 e 6, iniciou-se a etapa de montagem física do protótipo, conforme apresentado na Figura 7. Nessa etapa, ocorreram os ajustes manuais necessários para avaliar a funcionalidade do conjunto do pedal e da bomba de água.
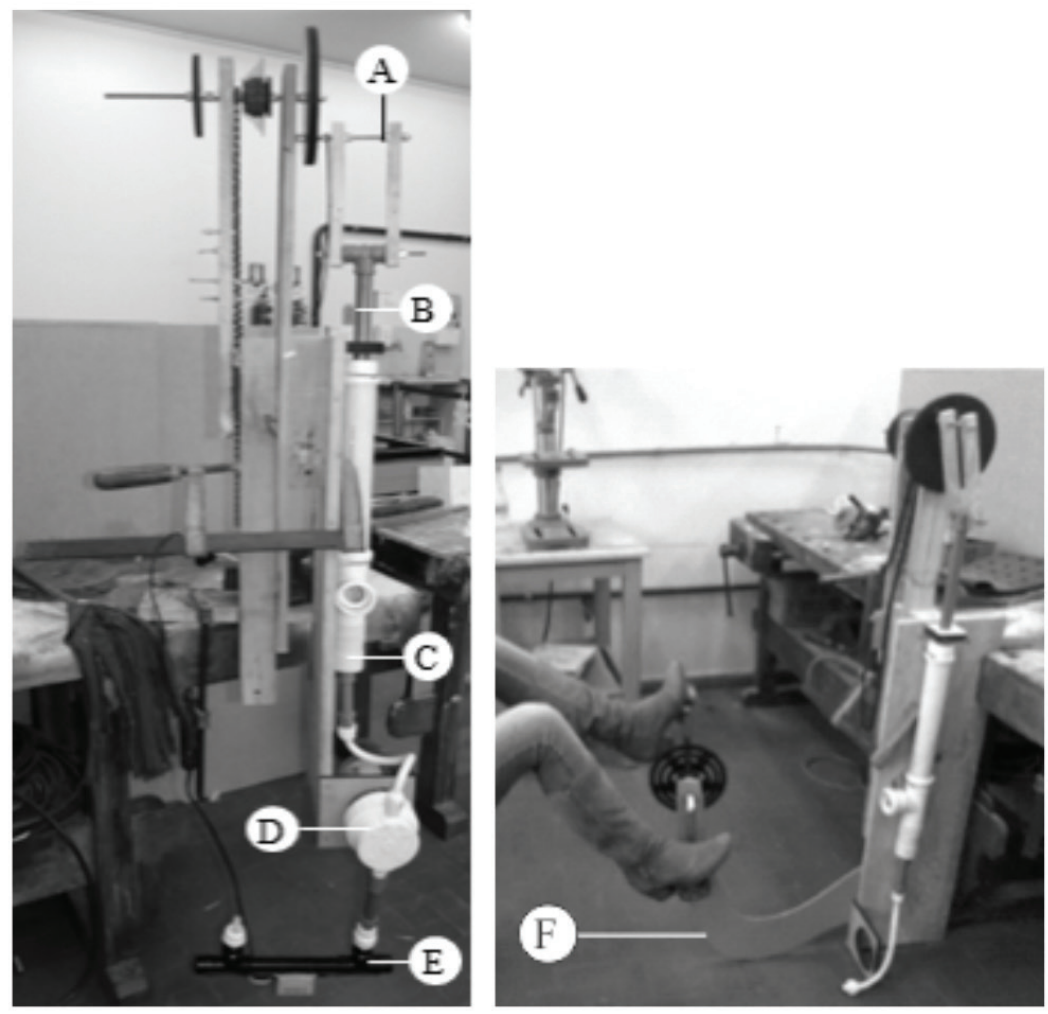

A Figura 7A mostra o sistema de mancal e de movimento da bomba. A Figura 7B mostra o eixo de movimento da bomba. A Figura 7C mostra o corpo da bomba. A Figura 7D mostra o filtro de água com elemento filtrante à carvão mineral. A Figura 7E mostra a lâmpada germicida e a Figura 7F mostra o braço de apoio para o pedal de acionamento da bomba; a Figura 7F mostra o braço de apoio do pedal. A sequência construtiva e de ajustes é apresentada na Figura 8. Para essa sequência foram testadas todas as peças fabricadas via corte laser.
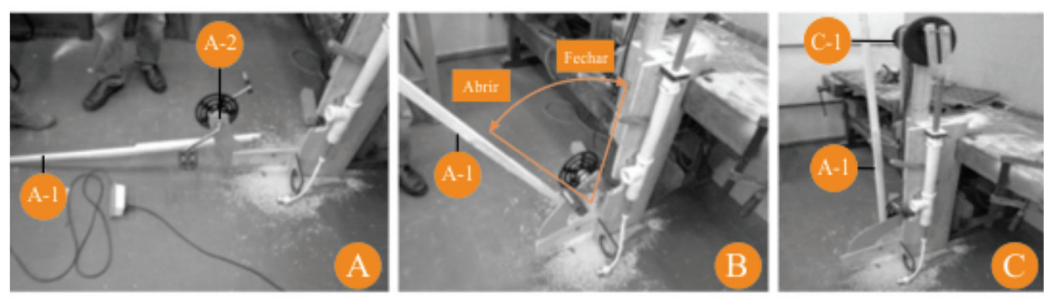

Observa-se na Figura 8A a coluna guia (A-1) e o dispositivo de pedais (A-2) utilizados para a movimentação da bomba e da geração de energia. 
A Figura 8B apresenta a coluna guia (A-1) em processo de fechamento. A Figura 8C mostra a coluna guia (A-1) fechada e o posicionamento da engrenagem (C-1) do movimento da bomba de água. Os ajustes finos foram realizados por meio de processos manuais. A utilização desses processos manuais, no contexto da prática pedagógica de oficina, possibilitou a integração dos conhecimentos teóricos adquiridos com as adequações de projeto. Após, validada a melhor alternativa, iniciou-se a etapa de desenvolvimento virtual em software de projeto.

\section{Desenvolvimento virtual do projeto}

A partir do estudo funcional, realizado no protótipo construído, foram geradas as alternativas para melhor dispor os componentes no produto final. Neste sentido, a Figura 9 apresenta a modelagem virtual gerada pelo software Inventor®.
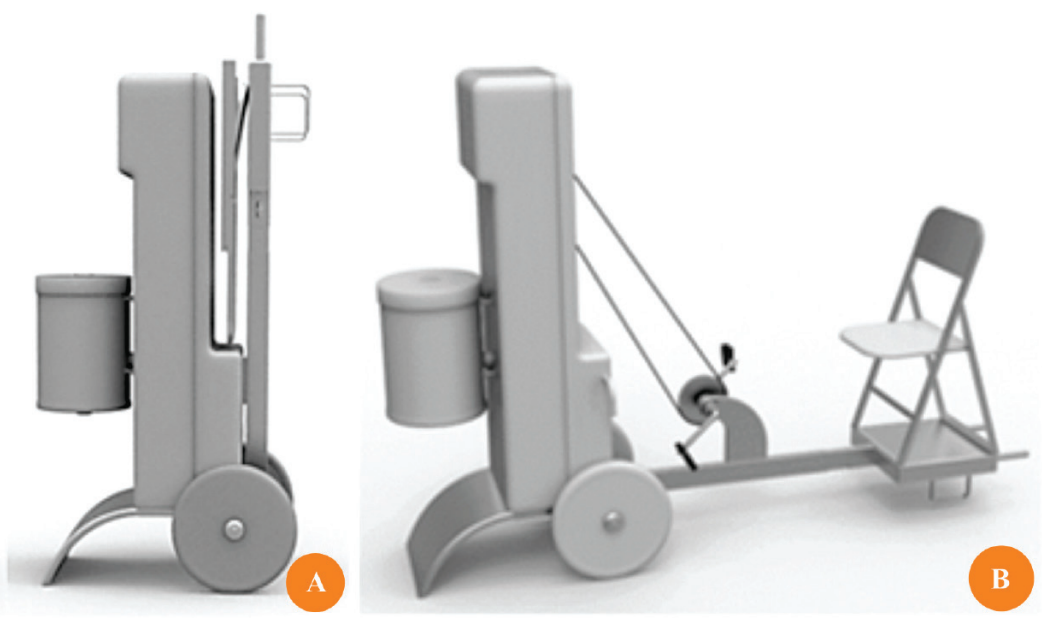

A Figura 9A mostra uma vista lateral do produto em sua posição fechada e pronto para o transporte pelo usuário. A Figura 9B mostra uma vista lateral do produto armado e pronto para a sua utilização principal, ou seja, realizar o processo de filtragem. Cabe salientar que o produto em escala real possui dimensões de largura $525 \mathrm{~mm}$, altura de $1526 \mathrm{~mm}$ e comprimento de 2179 $\mathrm{mm}$, em estado aberto. Observa-se, a construção estética orientada pela necessidade funcional e estrutural dos dispositivos internos, construídos na modelagem física. Essa percepção estética é fundamentada no momento em que o acadêmico absorve os conceitos levantados, de forma objetiva, durante a fabricação e ensaio das alternativas prototipadas.

\section{CONSIDERAÇÕES FINAIS}

O delineamento experimental dessa pesquisa visou a demonstrar a potencialidade do emprego de tecnologias construtivas para a discussão da escolha da melhor alternativa de projeto durante o ensino didático de Design. Podemos inferir, diante da facilidade e rapidez do uso do corte a laser para a fabricação de peças, que ao integrar essa técnica construtiva na seleção de alternativas, poderemos ser mais objetivos e éticos. Entretanto, esse fato é consolidado quando fabricamos um maior número de alternativas de projeto. A fabricação ampliada possibilita uma maior compreensão sobre os fatores funcionais e estruturais orientadores para
Figura 9. Modelagem virtual do equipamento. 
a escolha da alternativa final. Desse modo, o acadêmico tende a se sentir mais seguro sobre os motivos da seleção da respectiva forma e dos aspectos analisados. Embora a seleção de alternativas seja fortemente abordada por um viés teórico, o presente trabalho demonstrou que devemos ampliar o aspecto dessa seleção por parâmetros técnico-científicos.

Assim, os resultados apontados na Tabela 3, demonstram que é necessário conhecer os parâmetros funcionais dos equipamentos utilizados na prática construtiva. Nesse sentido, e diante dos resultados apresentados na Tabela 3, se julgássemos que a impressão 3D fosse a melhor alternativa para a construção das engrenagens - Figura 5 -, incorreríamos na ampliação do tempo de processo e da tomada de decisão final. Pelo contrário, ao utilizarmos a tecnologia de corte a laser, poderemos ampliar a fabricação do número de alternativas desde que sejam peças de corte plano. Dessa forma, estaremos reduzindo a possibilidade da decisão da melhor alternativa ser orientada por afinidades subjetivas, aleatórias ou pessoais.

As barreiras para aplicação pedagógica do uso de práticas construtivas residem, primeiramente, na sensibilização gerencial, que deve ser orientada para o investimento em estruturas e ferramentas tecnológicas. Em segundo lugar, a necessidade de integração da tecnologia ao processo criativo em disciplinas de projeto e, em terceiro lugar, necessita-se de uma estrutura que proporcione aos professores e acadêmicos o acesso a essa estrutura. Todavia, é preciso integrar, também, pessoal técnico capacitado para apoiar esse processo.

Diante do estudo de caso apresentado, pode-se concluir que a utilização de tecnologias construtivas de rápido resultado, como o corte a laser, tende a estimular o processo decisório do acadêmico. Assim, com a utilização desse processo, podemos apontar por fundamentos objetivos o passo-a-passo que direcionou a escolha da alternativa final.

\section{REFERÊNCIAS}

ABRAMOVITZ, J.; REBELLO, L. H. B. Metodologia do projeto. Rio de Janeiro: UniverCidade/NPD, apostila de aula, 2002.

ALVARES, M. R. O ensino do design: a interdisciplinaridade na disciplina de projeto em design. Dissertação (Mestrado em Engenharia de Produção) - Universidade Federal de Santa Catarina, Florianópolis, 2004

ASHBY, M. F.; JOHNSON, K. The art of materials selection. Materialstoday, Oxford, v. 6, n. 12, p. 24-35, dez. 2003.

BACK, N.; OLIGARI, A. DIAS, A.; SILVA, J. C. Projeto integrado de produtos: planejamento, concepção e modelagem. Barueri: Manole, 2008.

BAXTER, M. Projeto de produto: guia prático para o design de novos produtos. 2. ed. São Paulo: Edgard Blücher, 2000.

BITTENCOURT, A. C. P. Desenvolvimento de uma metodologia de reprojeto de produto para o meio ambiente. Dissertação (Mestrado em Engenharia Mecânica) Universidade Federal de Santa Catarina, Florianópolis, 2001.
BOMFIM, G. A. Metodologia para o desenvolvimento de projetos. João Pessoa: Universitária/UFPB, 1995.

BONSIEPE, G. Metodologia experimental: desenho industrial. Brasília, DF: CNPq/ Coordenação Editorial, 1984.

BOOTHROYD, G.; ALTING, L. Design for assembly and disassembly. CIRP Annals - Manufacturing Technology, v. 41, n. 2, p. 625-636, 1992

CAMARGO, F. R. Modelo para análise e seleção de alternativas na etapa conceitual de projeto. Dissertação (Mestrado em Engenharia Mecânica e de Materiais) - Universidade Tecnológica Federal do Paraná, Curitiba, 2007.

FLORIO, W. Modelagem paramétrica, criatividade e projeto: duas experiências com estudantes de Arquitetura. Gestão \& Tecnologia de Projetos, v. 6, n. 2, dez. 2011.

FORTI, F. S. A. Uma avaliação do ensino da prototipagem virtual nas graduações de design de produto do Estado do Rio de Janeiro. Dissertação (Mestrado em Engenharia Civil) - Universidade Federal do Rio de Janeiro, Rio de Janeiro, 2005. 
KAMINSKI, P. C. Desenvolvendo produtos: planejamento, criatividade e qualidade. Rio de Janeiro: Livros Técnicos e Científicos, 2000.

KELLEY, T.; LITTMAN, J. As 10 faces da inovação: estratégias para turbinar a criatividade. Rio de Janeiro: Elsevier, 2007.

LÖBACH, B. Desenho industrial: base para configuração dos produtos industriais. São Paulo: Edgar Blücher, 2000.

PERTENCE, A. E. M.; SANTOS, D. M. C.; JARDIM, H. V. Desenvolvimento de modelos didáticos para o ensino de desenho mecânico utilizando o conceito de prototipagem rápida. CONGRESSO BRASILEIRO DE EDUCAÇ̃̃O EM ENGENHARIA, 29., 2001, Porto Alegre. Anais... Belo Horizonte: Universidade Federal de Minas Gerais, 2001. Disponível em: <http://www.abenge.org. br/CobengeAnteriores/2001/trabalhos/ NTM047.pdf>. Acesso em: 9 nov. 2015.
PLATCHECK, E. R. Metodologia de ecodesign para o desenvolvimento de produtos sustentáveis. São Paulo: Atlas, 2012.

PRODANOV, C. C. Metodologia do trabalho científico: métodos e técnicas da pesquisa e do trabalho acadêmico. Novo Hamburgo: Feevale, 2009.

ROOSEMBURG, N.; EEKELS, N. Product design: fundamentals and methods. West Sussex: Wiley, 1996.

SANTOS, E. S. Um sistema informacional e perceptivo de seleção de materiais com enfoque no Design de calçados. 2005. Dissertação (Mestrado Profissionalizante em Engenharia Ambiental e Tecnologias Limpas) - Universidade Federal do Rio Grande do Sul, Porto Alegre, 2005.

VOLPATO, N. Prototipagem rápida (tecnologias e aplicações). 1. ed. São Paulo: Blucher, 2007.
Luis Henrique Alves Cândido candido@ufrgs.br

Suzane Miritz

ndsm@ufrgs.br

Jéssica do Canto Pureza ndsm@ufrgs.br 